https://www.journal-imab-bg.org

Review article

\title{
SOCIAL STATUS AND GUT MICROBIAL DYSBIOSIS IN ADULTS AND CHILDREN WITH AUTISM SPECTRUM DISORDERS: A PRO- FOUND REVIEW OF THE AVAILABLE LITERA- TURE SOURCES
}

\author{
Sevginar Ibryamova ${ }^{1}$, Veselin Petkov ${ }^{2}$, Tsveteslava Ignatova-Ivanova ${ }^{1 *}$, Georgi \\ Kolev $^{3}$ \\ 1) Department of Biology, Faculty of natural sciences, Shumen University, Bul- \\ garia \\ 2) Department of Geography, regional development and tourism, Faculty of \\ natural sciences, Shumen University, Bulgaria \\ 3) Department Social work, Faculty of Education, Shumen University, Bulgaria.
}

\begin{abstract}
:
Autism is a complex disorder without a specific diagnosis, so the disease is defined by its specific characteristics described in the literature as cognitive defects, social, communication and behavioral problems, repetitive behaviors, unusual sensitivity to stimuli such as noise, restricted interests, and self stimulation. There are many models in the literature explaining the biology of autism, which are based on genetics, immunity, various environmental factors and diet. There is a lot of literature data that people with Autism Spectrum Disorders (ASD) often have gastrointestinal problems that also affect their behavior. ASD suffer developmental disabilities from an early age, which can be both physical and psychological. Often people suffer these problems even throughout their lives. This review aims to provide basic information on definitions, historical data, diagnostic methods, behavioral etiology, gastrointestinal and social problems in adults and children with ASD.
\end{abstract}

Keywords: Autism, gastrointestinal problems, social problems, probiotics, prebiotics,

\section{INTRODUCTION}

In the recent years, there was a tremendous increase in the number of scientific publications in peer-reviewed journals on the problems of people with autism (ASD), which has made patients and medics much more informed about the problem. Autistic Syndrome Disorders (ASD) include many social problems such as behavioral abnormalities and nervous system problems, genetic changes, and gastrointestinal problems that recur throughout life (Autism and Developmental Disabilities Monitoring Network Surveillance Year 2008 Principal Investigators and CDC, 2012) [1]. The term "autism spectrum disorders" (ASDs), which is defined by various experts in this field, reflects the wider range of clinical manifestations that today define autism [2]. ASDs represent 3 of the pervasive develop- mental disorders defined in the Diagnostic and Statistical Manual of Mental Disorders, Fourth Edition (DSM-IV), 3 and the newer Diagnostic and Statistical Manual of Mental Disorders, Fourth Edition, Text Revision (DSM-IV-TR ) 4: autistic disorder (AD), Asperger syndrome (AS), and pervasive developmental disorder - not specially specified (PDD-NOS). There is evidence that even in the DSM-IVTR categories, additional subtypes can be defined. There are much clinical evidence that people with autism have gastrointestinal (GI) problems, which scientists explained by decreased bifidobacteria and increased Clostridium spp., Desulfovibrio spp., Sutterella spp. and/or Veillonellaceae [3]. Studies to date have shown that genetic predisposition mainly plays a role in the onset of the disease but is also influenced by the various environmental factors to which the mother is exposed during pregnancy [4]. Usually, the first symptoms appear before and around 3 years of age and today, there is still no drug treatment for ASD. Initially, there are disorders in nervous development, social relationships and communications, limited communication abilities and others. It all starts before the age of 3 . Autism is thought to affect the processing of information in the brain by changing the way nerve cells and their synapses connect, but there is still no clear evidence that this is true. The other two known disorders (ASD) are Asperger's syndrome, in which there are no delays in cognitive development and language - called atypical autism and PDD-NOS - when no comprehensive developmental disorders are indicated. ASD can have a negative impact and destroy families who are not well informed about how to burden themselves with a child with autism, as communication with such children is very difficult [4]. According to data from parents of children with autism, there are many problems related to the time of eating and limiting the type of food they consume. This problem, called selective eating, can lead to so-called inadequate nutrition and lead to a limited diet. This is a serious problem facing families of chil- 
dren with ASD. More and more attention was paid to the possible inclusion of a variety of foods in people suffering from ASD as one of the methods of treatment and change in the behavior of ASD. In these treatments, the effect of different diets on physiological and behavioral changes in children with ASD has been observed [4].

The main goal of this review is to summarize the literature data on social and gastrointestinal problems in adults and children with autism, which can be used to better understand, diagnose and follow diets.

\section{Social and GI problems of adults with autism}

There are many problems in predicting autism due to the very wide range of cognitive, linguistic, social and behavioral functions associated with the condition. Despite many studies in adolescents or adults with autism, the prognosis information remains inconclusive due to the heterogeneity of the subjects, the variability of the baseline measures used, and other fundamental problems of the research methods. The earliest studies are largely unrealistic and involved people of different ages, making it difficult to determine the outcome for a particular age structure [5]. In 1973, Kanner [5] reported 96 individuals, mostly in their twenties and thirties. Most of them lived with their families in closed communities, government institutions or psychiatric hospitals. As a result, more than half of the people with ASD had better communication skills, and just over half of this group function relatively well. Of all who were surveyed, 11 people started work, and 1 has graduated from college. Seven were single and one was married with a child. The first systematic outcome studies were conducted by Rutter and his colleagues in the $60-70$ years. The study was conducted on 38 individuals aged 16 years. Most were hospitalized for a long time, 7 lived with their parents, 4 attended day centers, and 3 were living in residential communities; only 3 had paid jobs. Overall, 14\% were reported to have made a "Good" social adjustment; $25 \%$ were rated as "Fair" and 61\% as "Poor". Lotter (1974) [5] studied 29 individuals aged 16 to 18 years with ASD. Of these children, 22 dropped out of school, half were hospitalized, and only one was employed. Five people were housed in centers, and only 2 lived in families. Fourteen per cent were rated as having a "Good" outcome; $23 \%$ as "Fair", and $63 \%$ as "Poor" or "Very Poor". In 1987, Gillberg and Steffenburg [22] reported on a group of 23 individuals aged 16 or over living in Sweden. Only one person was found to be fully self-supporting; of the remainder, $48 \%$ were rated as having a "Fair" outcome and the same proportion as "Poor" / "Very Poor". Kobayashi, Murata, and Yashinaga and colleagues (1992) used a postal survey to track 201 people aged 18 to 33 in Japan over a period of fifteen years. The survey showed that four males have died, 5\% attend school or college; $20 \%$ were employed, mostly in the food industry. All but 3 of those at work lived with their parents, and 2 had their own homes. All were single. Twenty-seven per cent of the group was rated as having a Good or Very Good outcome on the basis of their adaptive skills. The same proportions were rated as Fair, whilst $46 \%$ were rated as Poor or Very Poor. A telephone survey by Ballaban-Gil, Rapin,
Tuchman, and Shinnar (1996) found that of the 45 adults initially diagnosed as children with ASD, more than half were housed in homes, and only one lived alone. Three people had died, and eleven percent were working. Behavioral problems were reported in all people, and only in 3, no social conflicts were identified. Estimates of changes in IQ levels from child to adulthood indicated that cognitive functioning remained very stable, and only $18 \%$ showed a marked change in their IQ. When change did occur, this usually indicated an improvement rather than a decline in intellectual abilities. Comparisons between studies need to be treated with caution because of the differences in sample selection and in the measures used. Most investigations have involved relatively small groups of subjects, diagnostic criteria were sometimes imprecise and/or the quality of data on early intellectual functioning was poor. Overall judgments of whether the outcome is Good, Fair or Poor also tend to be based on variable criteria. Nevertheless, the one consistent conclusion from these reports is that a positive prognosis in adulthood is highly dependent on IQ - very few individuals with an IQ below 50 achieve good functioning in adulthood. The result is bad for those who fail to speak for about 6 years. However, even among people with a higher IQ, the result is very variable. Rumsey, Rapoport, and Sceery (1985) [5] conducted a study with 14 men between the ages of 18 and 39, all of whom met the DSM-III criteria for autism. Verbal and/or IQ scores are 60 or higher. Socially, the whole group continued to face significant difficulties. Only one person had friends, and four were self-employed. More than $70 \%$ are highly dependent on their parents, and one is hospitalized. Similar data were reported by Tantam (1991) [5] in a study of 46 individuals (mean age 24 years) with Asperger's syndrome. Venter, Lord, and Schopler (2002) [5] studied 22 individuals over the age of 18 years who had an IQ of $60+$ in preschool. About a third were employed, but jobs were very low rated. Most were employed in special training programs, and 3 did not work. Only 4 individuals lived independently. However, Szatmari and his colleagues [5] reported better results among a group of 12 men and 4 women (all 17 years or older; mean IQ> 90). Half attended college or university, and more than a third worked fulltime. Half were completely independent, although some of them still live in a family environment. One member of the group was married. Most recently, Mawhood and colleagues [5] followed 19 men with autism (average WAIS PIQ 83) who were initially diagnosed between 4 and 9 years of age. All observed had long-term social and communication problems. Almost half lived in isolation, only 3 were independent. Only 3 people, which is (16\%) have achieved good behavioral results; 2 people $(10 \%)$ remain with minor disabilities, and 14 (74\%) continue to show significant disabilities.

In recent years, many studies have reported gastrointestinal problems in individuals with ASD, often with severe symptoms. Adam and colleagues report a strong relationship between the symptoms of the gastrointestinal tract and the severity of autism. These results were also con- 
firmed by Tomova et al. in a more recent study [6]. There are various gluten-free and casein-free diets (GFCF) that are offered to relieve symptoms, but to date, there are insufficient evidence of their effect on gut health. Many studies reported relieving gastrointestinal problems and/or improving behavioral traits with GFCF, but the links between a restricted diet and symptoms are not always obvious and clear [6]. Some studies evaluating mood and behavior showed significant behavioral improvements, while others did not report any improvement after treatment with such diets. The intestinal microbiota in humans affects health and is strongly influenced by the diet. Several studies have focused on GM modulators, such as probiotics in ASD, but there is a discrepancy in the estimated parameters [7]. Parracho et al. focused on behavioral characteristics, showed improvement after administration of a probiotic with Lactobacillus Plantarum WCSF1 in children with ASD, while Adam et al. (2018) show significant positive differences in the levels of organic acids in the feces in individuals with ASD taking probiotics [8]. Metabolic changes were also observed by Kaluzna-Czaplinska and $\mathrm{B}^{3}$ aszczyk (2012), who, after 2 months of oral addition with L. acidophilus (strain Rosell-11), found a significant decrease in the level of D-arabinitol (DA) -positive modification due to its association with the pathogenic Candida spp. [8]. In addition, Tomova [6] and colleagues evaluated the effect of a 4-month mixed administration of probiotics (Children Dophilus) on the genetically modified composition in children with ASD, and they were able to show a change in the Bacteroidetes / Firmicutes ratio, i. increase in bifidobacteria. Prebiotics are food supplements that are selectively absorbed and metabolized by local beneficial bacteria, thus positively modulating the gastrointestinal tract. Their effects on autism have not been well studied. The prebiotic B-GOS $®$ (galactooligosaccharide) has been shown to affect faecal microbiota composition and metabolic profile using an in vitro fermentation system that mimics autistic colon conditions [9]. Its effect in vivo, especially taking into consideration the different dietary approaches that children with ASD can follow, has not been studied. In the study of Grimaldi et al. (2018) [9], significant behavioral improvements have been reported after the addition of B-GOS $®$ in children with ASD. This is the first time that the effects of the diet on the composition and metabolism of the gastrointestinal tract, their potential relationship to gastrointestinal discomfort and the effect of a prebiotic supplement have been studied in the context of autism.

Significant differences in microbiota and metabolism were found at the base level when diets were compared. This may indicate that the diet affects the condition and composition of the intestinal tract. Prior to the intervention of the prebiotic B-GOS $\AA$, the intervention evaluated the nutritional impact of gluten-free diets (GFCF), and the results showed a significant deficiency in vitamin D intake in children on unlimited diets [10]. These findings are supported by recent studies in Spain, where a group of 105 autistic people on a restricted diet consumed fewer carbohydrates and more legumes and vegetables [11]. The new in this study is the intake of low doses of vitamin D, which is considered an active neurosteroid during brain development, and its deficiency is a potential risk factor for those diagnosed with ASD [12]. According to the literature, there is a hypothesis called "opioid surplus theory". According to it, the metabolism of gluten and casein leads to excessive production of opioid compounds, causing side effects such as constipation and behavioral problems [13]. Therefore, gluten- and casein-free diets have been suggested to alleviate such problems, but [10] studies have shown that in both restricted and unrestricted diets, the results of abdominal pain are under "one". In addition, other studies reported that individuals with ASD tolerate the introduction of gluten and casein in their diet, questioning the efficacy of the GFCF diet [14]. Currently, autism analyzes mainly focus on identifying potential biomarkers in terms of intestinal composition, usually comparing faecal samples from siblings and non-autistic controls [15]. The results of these studies show several genera present in larger quantities in ASD, such as Bacteroides, Roseburia, Akkermansia, Hespellia spp. and others with smaller amounts, such as Prevotella and bifidobacteria [16]. There are no studies evaluating the effect of GFCF dietary approaches on GM in ASD individuals. Roberta Grimaldi et al. (2018) [10] also show that fluctuations between Bacteroides spp. and propionate in faecal samples from children with ASD on gluten-free diets. SCFAs have been shown to affect the CNS, and in particular intraventricular propionate injection in mice has been shown to cause autistic behavior. Data provided by Grimaldi et al. (2018) [10] show the potential role of GM in the production of metabolites that may be associated with autistic traits. However, the actual effect of SCFAs produced in the gut on the CNS is not entirely clear, as the concentrations that can cross the blood-brain barrier (BBB) may be small.

\section{Autism in children}

It is extremely important for all families where a child with autism lives to have accurate information about future developments that would be helpful in dealing with this problem. The exact etiology of ASD is unknown. In 1943, Leo Kanner, a psychiatrist at Johns Hopkins University, first described autism in a small group of children characterized by complete distance from other people [5]. In 1944, Hans Asperger, an Austrian pediatrician who was unaware of Kanner's work, published an article, that described children who demonstrated symptoms similar to those of Kanner's patients, with the exception that verbal and cognitive skills were higher. The term "infantile autism" first appeared as a diagnostic label in the Diagnostic and Statistical Manual of Mental Disorders, Third Edition (DSMIII) [2]. After expanding of the diagnostic criteria, the terminology had changed. After many studies published in recent years, it is clear that the prevalence of ASD in Europe and North America is approximately 6 per 1000 [5]. In 2000, the Centers for Disease Control and Prevention organized the Autism and Developmental Disabilities Monitoring Network, a multisite, records-based surveillance 
program, to study the prevalence of ASDs. The network uses systematic screening of developmental evaluation records for autistic behaviors rather than depending on a medical or educational diagnostic label of an ASD. In 2007, the network reported ASD rates for 8-year-old children ranging from 1 in 303 to 1 in 94 for 2 time periods (2000 and 2002) in a total of 14 sites in the United States; the average rate was 1 in 150 or 6.6 per 1000 8-yearolds. These studies reflect a 10 -fold increase in studies published half a century ago [2]. Although there are many theories about the etiology of ASD, very little is known about the pathophysiology of this disease. Gastrointestinal problems and behavior deviations have often been reported in children with ASD. There is still no clear explanation for the link between gastrointestinal problems and ASD, however food intolerance is thought to play a major role in ASD [17].

It is extremely important for all families, where a child with autism lives, to have accurate information about future developments that would be helpful in dealing with this problem. The exact etiology of ASD is unknown. In 1943, Leo Kanner, a psychiatrist at Johns Hopkins University, first described autism in a small group of children characterized by complete distance from other people. In 1944, Hans Asperger, an Austrian pediatrician who was unaware of Kanner's work, published an article that described children who demonstrated symptoms similar to those of Kanner's patients, with the exception that verbal and cognitive skills were higher. The term "infantile autism" first appeared as a diagnostic label in the Diagnostic and Statistical Manual of Mental Disorders, Third Edition (DSM III) [2]. After expanding the diagnostic criteria, the terminology changes. After many studies published in recent years, it is clear that the prevalence of ASD in Europe and North America is approximately 6 per 1000 [5]. In 2000, the Centers for Disease Control and Prevention organized the Autism and Developmental Disabilities Monitoring Network - a multisite, records-based surveillance program, to study the prevalence of ASDs. The network uses systematic screening of developmental evaluation records for autistic behaviors rather than depending on a medical or educational diagnostic label of an ASD. In 2007, the network reported ASD rates for 8-year-old children ranging from 1 in 303 to 1 in 94 for 2 time periods (2000 and 2002) in a total of 14 sites in the United States; the average rate was 1 in 150 or 6.6 per 10008 -year olds. These studies reflect a 10 -fold increase in studies published half a century ago [2].

Although there are many theories about the etiology of ASD, very little is known about the pathophysiology of this disease. Gastrointestinal problems and behavior have often been reported in children with ASD. There is still no precise explanation for the relationship between gastrointestinal problems and ASD; it is suggested that food intolerance plays a major role in ASD [17].

Colonization of the gut with microorganisms is known to begin at birth, but the sequence and composition of an individual's microbial community depend on many factors, such as age, diet, genetic setup, gender, geographic location, and health. The composition and role of the intestinal microbiota and its effects on human health have been well studied, with the exception of the Indian population [18]. The main observation from these studies shows that the genera Prevotella and Megasphaera predominate in the Indian population [19], which is due to the typical Indian diet rich in carbohydrates [18]. It is assumed that this feature is determined by diet, diet, environment and ethnicity [74]. Food intolerance is thought to play a major role in ASD [18-19]. Many studies have shown that gluten-free casein diets reduce gastrointestinal disorders and improve the behavior of individuals with ASD [19]. The exact percentage of people suffering from gastrointestinal (GI) problems shows variability in different studies and depends on the age of the individuals studied. What all research demonstrated was that GI problems are common in autism. In one study of 172 children with ASD, 24\% had at least one gastrointestinal symptom, such as diarrhea, constipation, bloating and/or gastroesophageal reflux disease (GERD). In another study of 160 children with ASD, it was found that $59 \%$ had GI dysfunction with diarrhea or unformed stools, constipation. A study of 51 children with ASD compared with 40 typical control groups aged 3-15 years found that $63 \%$ of children with autism reported moderate or severe chronic diarrhea and/or constipation, compared with $2 \%$ of control children. In summary, these studies show that GI symptoms are common in autism. Recent studies report the important role of the intestinal microbiota in brain function, which is of great importance for human health [20]. On the other hand, intestinal dysbiosis has an adverse effect on human health [21].

There are also evidence that if mothers become overweight during pregnancy, it can lead to problems in the child's intestinal microbiome and affect his neurodevelopment and social behavior. The term gut-brain axis has been defined as the biochemical signalling communication between the gastrointestinal tract and the central nervous system. Many scientific publications showed that dysbiosis causes changes in intestinal metabolites that are important for cytokines and neuroactive compounds. This can lead to severe mental disorders [21]. Many animal experiments have also shown that dysbiosis affects their behavior [22]. There are currently approximately 1.72 million cases of ASD in India, and the number is gradually increasing with improvements in early diagnosis methods. In the study by Pulikkan et al. (2018) [17], an investigation of the intestinal microbiota was performed in 30 children with ASD by comparison with 24 healthy children from the Indian population by sequencing the V3 hypervariable region of the $16 \mathrm{~S}$ rRNA gene. One unique aspect of this study is that both ASD and healthy children were subjected to the same diet, unlike previous studies in which children with ASD consumed a gluten-free diet. Children with ASD experience GI-related problems, such as constipation and diarrhea, while healthy children do not show such problems. The change in the amount of Lactobacillus in healthy children and children with ASD was reported. Lactobacillus volumes were found to be 3232 times higher in children with ASD than in healthy children. In addition, higher levels of Lactobacillus are observed in most chil- 
dren with ASD compared to healthy children, indicating that the observed high levels of Lactobacillus are not uncommon [17]. In recent years, it has been suggested that there is a link between the species diversity of human microorganisms and recently were identified relations between the microbiota of the human gut and physiological and immunological diseases [23]. The intestinal microbiota also influences behavior [23]. Mechnikov's catchphrase "The great variety of microorganisms inhabiting the human gastrointestinal tract is determined by a person's spiritual and physical health" is well known.

To date, more and more data on symptoms such as behavioral changes and gastrointestinal complications are regarded as common and well-established features of ASD, making its etiology better understood [24]. Studies based on the 16S rRNA gene sequence have shown dysbiosis of the intestinal microbiome in children with ASD in the American and European populations [25]. The change in the intestinal flora in ASD is also associated with a reduction in some types of bacteria such as Sutterella, Akkermansia, and Fusobacterium. The proportion of Firmicutes was found to be significantly higher in cases of ASD, as confirmed by earlier reports, where a higher proportion of Firmicutes as compared to Bacteroidetes was associated with intestinal microbiome dysbiosis and intestinal diseases [25]. Several recent reports also suggest that intestinal microbial dysbiosis may modulate human behavior and also play a role in the development or presentation of ASD-related symptoms [26]. Prevotellaceae has been found to be most common in the faecal microbiota of healthy children [17]. A similar study was done in a recent report on the Indian population [27]. In contrast to the Indian population, Bacteroidaceae microorganisms predominate in the populations of America and Europe [27]. These differences seem to be mainly due to differences in the geographical location and diet between the two populations. Lactobacillus (family Lactobacillaceae), Bifidobacterium (family Bifidobacteraceae), Megasphaera, and Mitsuokella (Veillonellaceae) were found in large quantities in children with ASD compared to healthy children. The Veillonellaceae family predominates in carbohydraterich gluten-containing diets [28-29]. When comparing children of Indian descent and American children with ASD, it was found that in Indian children with ASD, the genus Megasphaera, Bifidobacterium, and Mitsuokella predominate, while in the intestinal microbiome of children with ASD in the US it is rich in genus Akkermansia, Ruminococcus, and Lactobacillus. These differences could be explained by the different eating habits and consumption of a gluten-free diet by children with ASD. Interestingly, Prevotella is absent in children with ASD from both populations. Most notably, Lactobacillus appears to be the only common genus in both populations that shows significantly greater abundance in children with ASD than in healthy children. On the other hand, although Lactobacillus was present in large amounts, the authors found no association between Lactobacillus and autism. Bacterial species belonging to the genus Lactobacillus produce lactic acid, known as lactic acid bacteria (LAB) and have been the subject of much research in recent years due to its probiotic nature. Lactobacillus is a major inhabitant of our intestines and is considered very important for the health of the intestinal microbiota in infants, which is primarily because their diet is rich in lactate from milk. The amount of these bacteria decreases with age as the diet becomes more varied and decreases based on milk consumption [17].

Both Lactobacillus and Bifidobacterium are considered probiotic bacteria with a beneficial role in human health. The immunomodulatory properties of LAB, including Bifidobacterium, have been extensively studied in recent years. A study by Cvijin et al. (2015) showed that a higher proportion of Lactobacillus leads to the inhibition of an immune modulator enzyme IDO1 (indoleamine 2,3dioxygenase), which ultimately affects the Th17 cell functioning and leads to the atopic dermatitis condition [30]. Another study reports a higher proportion of IL-6, IL-10, TNF-á (pro-inflammatory cytokine), and certain chemokine production by NF-êâ pathways in the intestinal epithelium cells with a Lactobacillus strain [30]. Higher levels of some pro-inflammatory cytokines (IL-6, IL-12p40) have been associated with impaired communication, abnormal behavior, and atopic dermatitis by disrupting the blood-brain barrier in ASD. Influence on the behavior of the patients due to different types of lactobacilli was shown in an experiment. In observations of humans with $\mathrm{ASD}, L$. reuteri has been shown to return ASD-like behavior. L. ruminis has been shown to have immunomodulatory activity and is the most common species in ASD cases in this study [31]. The same study showed that short-chain fatty acids (SCFA) are important for the intestinal microflora in ASD [31]. SCFAs are bacterial metabolites derived from intestinal microbes that play a role in the immune system, brain function, and behavior [32]. Dysbiosis in the intestinal microbiota is thought to lead to an imbalance in the levels of SCFAs in the circulatory system, which can lead to increased intestinal permeability, detrimental to children with ASD. Also, the great species diversity of the Lactobacillaceae and Bifidobacteriaceae families is associated with a reduction in SCFA-producing bacteria [33]. Pulikkan et al. (2018) [17] showed that the reduction of some SCFA-producing microbes, such as Faecalibacterium and Roseburia in children with ASD, supports the hypothesis that lower SCFA levels in ASD lead to an imbalance in brain function and behavior. It has been proposed to introduce these strains as a probiotic to the affected children. This can help relieve common gastrointestinal problems. Other microbial families such as Veillonellaceae and Enterococcaceae have also been found to be higher in children with ASD [34]. These results are confirmed by a report by Oberc et al. (2015), which suggests their association with other gastrointestinal disorders such as Crohn's disease [35]. The probable reason for the presence of these species is the local omnivorous diet of children with ASD, in contrast to the gluten-free diet in other similar studies [29].

Gastrointestinal problems in children with autism can contribute to the severity of the disorder. Abdominal pain, constipation and/or diarrhea are unpleasant and are likely to lead to frustration, decreased ability to concen- 
trate, behavioral problems and possibly aggression and suicide, especially in children who cannot report their discomfort. These problems also lead to a reduced ability to learn to use the toilet on their own, which further complicates the child's social behavior and problems in raising him in old age [20]. The exact cause of gastrointestinal problems in children with autism is not yet clear. According to some authors, the problem is related to the unusual microflora or the excessive use of antibiotics, which can also change the species diversity in the gut. Frequently used oral antibiotics eliminate almost all normal intestinal microbiota, which plays an important role in the breakdown of plant polysaccharides, promotes gastrointestinal motility, maintains water balance, produces some vitamins and competes with pathogenic bacteria. Loss of normal intestinal flora can lead to overgrowth of pathogenic flora, which in turn can cause constipation and other problems and lead to severe dysbiosis. Finegold et al. 2002 [36] examined fecal samples from 13 children with late autism and 8 controls. The number and type of Clostridium and Ruminococcus in children with autism differ from the control children. Song et al. 2004 [37] found that cluster groups I and XI of Clostridium and Clostridium boltae had an average number of cells significantly higher than that of control children. Parracho et al. 2005 [38] levels of the group of bacteria Clostridium histolyticum are higher in children with ASD compared to typical children. C. histolyticum bacteria are recognized producers of toxins and may contribute to intestinal dysfunction. Finegold et al. 2002 [36] studied intestinal bacteria in children with autism and healthy children and found several differences, such as higher amounts of Bacteroides in the severely autistic group and higher levels of Firmicutes in healthy children. Finegold 2008 [39] suggest that 1) the recurrence of some children with autism after antibiotic treatment is caused by increased levels of Clostridium spores, 2) the frequency of autism is associated with prolonged exposure to Clostridium spores, and 3 ) the increase in cases Autism in a family is also as- sociated with contact with spores. Finegold also discusses the fact that propionate has severe neurological effects in rats, and Clostridia species are propionate producers. No human studies have been performed to determine whether the relative proportion of propionate is related to autism symptoms. Decreased activity of digestive enzymes has also been reported in children with autism. A study by Horvath and Perman 2002 [40] reported that 44 out of 90 (49\%) children with autism who underwent endoscopy (because they had significant gastrointestinal problems) had deficiencies in one or more disaccharide enzymes, especially lactase and maltase. They report that all children with low enzyme activity have loose stools and/or profuse flatulence. A recent study of children with autism and their first degree relatives found that $37 \%$ and $21 \%$, respectively, had increased intestinal permeability based on a lactulose/mannitol test, compared to $5 \%$ of normal subjects. They also found that autistic patients on a gluten-free, casein-free diet had significantly lower intestinal permeability [40].

\section{CONCLUSIONS}

Future research in children and adults with ASD should cover a larger population of people of different ages and regions around the world. It remains unclear whether the abundance of probiotic bacteria of the genus Lactobacillus is the cause or an effect of ASD. Many factors can influence the diagnosis, such as the influence of diet, habitat, and age. In addition, to better understand the function of the intestinal microbiome in people with ASD, the researcher will be necessary to understand the role and association of probiotics and other bacteria in different populations of humans.

\section{Acknowledgements}

The contributors express their gratitude for the funding by the project by Shumen University project RD-0867/25.01.2021 Department of Biology and National Program Young Scientists and Postdoctoral Students.

\section{REFERENCES:}

1. Prevalence of autism spectrum disorders-Autism and Developmental Disabilities Monitoring Network, 14 sites, United States, 2008. MMWR Surveill Summ. 2012 Mar 30;61(3):119. [PubMed]

2. Committee on Children With Disabilities. The Pediatrician's Role in the Diagnosis and Management of Autistic Spectrum Disorder in Children. Pediatrics. 2001 May;107(5): 12211226. [Crossref]

3. Li Q, Han Y, Dy ABC, Hagerman RJ. The Gut Microbiota and Autism Spectrum Disorders. Front Cell Neurosci. 2017 Apr 28;11:120. [PubMed]
4. Elder JH, Shankar M, Shuster J, Theriaque D, Burns S, Sherrill L. The gluten-free, casein-free diet in autism: results of a preliminary double blind clinical trial. J Autism Dev Disord. 2006 Apr;36(3):413-20. [PubMed]

5. Howlin P, Goode S, Hutton J, Rutter M. Adult outcome for children with autism. J Child Psychol Psychiatry. 2004 Feb;45(2):212-29. [PubMed]

6. Tomova A, Husarova V, Lakatosova S, Bakos J, Vlkova B, Babinska K, et al. Gastrointestinal microbiota in children with autism in Slovakia. Physiol Behav. 2015 Jan;138:179-87. [PubMed]

7. Gibson GR, Hutkins R, Sanders
ME, Prescott SL, Reimer RA, Salminen SJ, et al. Expert consensus document: The International Scientific Association for Probiotics and Prebiotics (ISAPP) consensus statement on the definition and scope of prebiotics. Nat Rev Gastroenterol Hepatol. 2017 Aug;14(8):491-502. [PubMed]

8. Coleman DM, Adams JB. Survey of vocational experiences of adults with Autism Spectrum Disorders, and recommendations on improving their employment. J Vocat Rehabil. 2018; 49(1):67-78. [Crossref]

9. Grimaldi R, Cela D, Swann JR, Vulevic J, Gibson GR, Tzortzis G, et 
al. In vitro fermentation of B-GOS: impact on faecal bacterial populations and metabolic activity in autistic and non-autistic children. FEMS Microbiol Ecol. 2017 Feb;93(2):fiw233. [PubMed]

10. Grimaldi R, Gibson GR, Vulevic J, Giallourou N, Castro-Mejia JL, Hansen LH, et al. A prebiotic intervention study in children with autism spectrum disorders (ASDs). Microbiome. 2018 Aug 2;6(1):133. [PubMed]

11. Mari-Bauset S, LlopisGonzalez A, Zazpe I, Mari-Sanchis A, Morales Suarez-Varela M. Comparison of nutritional status between children with autism spectrum disorder and typically developing children in the Mediterranean Region (Valencia, Spain). Autism. 2017 Apr;21(3):310322. [PubMed]

12. Cannell JJ. Vitamin D and autism, what's new? Rev Endocr Metab Disord. 2017 Jun;18(2):183-193. [PubMed]

13. Ly V, Bottelier M, Hoekstra PJ, Arias Vasquez A, Buitelaar JK, Rommelse NN. Elimination diets' efficacy and mechanisms in attention deficit hyperactivity disorder and autism spectrum disorder. Eur Child Adolesc Psychiatry. 2017 Sep;26(9):1067-1079. [PubMed]

14. Hyman SL, Stewart PA, Foley J, Cain U, Peck R, Morris DD, et al. The Gluten-Free/Casein-Free Diet: A Double-Blind Challenge Trial in Children with Autism. J Autism Dev Disord. 2016 Jan;46(1):205-220. [PubMed]

15. Strati F, Cavalieri D, Albanese D, De Felice C, Donati C, Hayek J, et al. New evidences on the altered gut microbiota in autism spectrum disorders. Microbiome. 2017 Feb 22;5(1):24. [PubMed]

16. Toh MC, Allen-Vercoe E. The human gut microbiota with reference to autism spectrum disorder: considering the whole as more than a sum of its parts. Microb Ecol Health Dis. 2015 Jan 28;26:26309. [PubMed]

17. Pulikkan J, Maji A, Dhakan DB, Saxena R, Mohan B, Anto MM, et al. Gut Microbial Dysbiosis in Indian Children with Autism Spectrum Disorders. Microb Ecol. 2018 Nov;76(4): 1102-1114. [PubMed]

18. Bhute S, Pande P, Shetty SA,
Shelar R, Mane S, Kumbhare SV, et al. Molecular Characterization and MetaAnalysis of Gut Microbial Communities Illustrate Enrichment of Prevotella and Megasphaera in Indian Subjects. Front Microbiol. 2016 May 9;7:660. [PubMed]

19. Kumbhare SV, Kumar H, Chowdhury SP, Dhotre DP, Endo A, Matto J, et al. A cross-sectional comparative study of gut bacterial community of Indian and Finnish children. Sci Rep. 2017 Sep 5;7(1):10555. [PubMed]

20. Saxena R, Sharma VK. A Metagenomic Insight into Human Microbiome: Its Implications in Health and Disease. In: Medical and Health Genomics. Editors: Kumar D, Antonarakis S. 1st edirion. Academic Press. 4 June 2016. Chapter 9. pp.107-117. [Crossref]

21. Vuong HE, Hsiao EY. Emerging Roles for the Gut Microbiome in Autism Spectrum Disorder. Biol Psychiatry. 2017 Mar 1;81(5):411-423. [PubMed]

22. de Theije CG, Koelink PJ, Korte-Bouws GA, Lopes da Silva S, Korte SM, Olivier B, et al. Intestinal inflammation in a murine model of autism spectrum disorders. Brain Behav Immun. 2014 Mar;37:240-7. [PubMed]

23. Maji A, Misra R, Dhakan DB, Gupta V, Mahato NK, Saxena R, et al. Gut microbiome contributes to impairment of immunity in pulmonary tuberculosis patients by alteration of butyrate and propionate producers. Environ Microbiol. 2018 Jan;20(1): 402-419. [PubMed]

24. Kelly JR, Minuto C, Cryan JF, Clarke G, Dinan TG. Cross Talk: The Microbiota and Neurodevelopmental Disorders. Front Neurosci. 2017 Sep 15;11:490. [PubMed]

25. De Angelis M, Francavilla R, Piccolo M, De Giacomo A, Gobbetti M. Autism spectrum disorders and intestinal microbiota. Gut Microbes. 2015; 6(3):207-13. [PubMed]

26. Vuong HE, Hsiao EY. Emerging Roles for the Gut Microbiome in Autism Spectrum Disorder. Biol Psychiatry. 2017 Mar 1;81(5):411-423. [PubMed]

27. Bhute S, Pande P, Shetty SA, Shelar R, Mane S, Kumbhare SV, et al. Molecular Characterization and Meta-
Analysis of Gut Microbial Communities Illustrate Enrichment of Prevotella and Megasphaera in Indian Subjects. Front Microbiol. 2016 May 9;7:660. [PubMed]

28. Ercolini D, Francavilla R, Vannini L, De Filippis F, Capriati T, Di Cagno R, et al. From an imbalance to a new imbalance: Italian-style glutenfree diet alters the salivary microbiota and metabolome of African celiac children. Sci Rep. 2015 Dec 18;5:18571. [PubMed]

29. Bonder MJ, Tigchelaar EF, Cai X, Trynka G, Cenit MC, Hrdlickova B, et al. The influence of a short-term gluten-free diet on the human gut microbiome. Genome Med. 2016 Apr 21;8(1):45. [PubMed]

30. Vujkovic-Cvijin I, Swainson LA, Chu SN, Ortiz AM, Santee CA, Petriello A, et al. Gut-Resident Lactobacillus Abundance Associates with IDO1 Inhibition and Th17 Dynamics in SIV-Infected Macaques. Cell Rep. 2015 Nov 24;13(8):1589-97. [PubMed]

31. Marlin BJ, Froemke RC. Oxytocin modulation of neural circuits for social behavior. Dev Neurobiol. 2017 Feb;77(2):169-189. [PubMed]

32. Sampson TR, Mazmanian SK. Control of brain development, function, and behavior by the microbiome. Cell Host Microbe. 2015 May 13;17(5):565-76. [PubMed]

33. Derrien M, van Hylckama Vlieg JE. Fate, activity, and impact of ingested bacteria within the human gut microbiota. Trends Microbiol. 2015 Jun;23(6):354-66. [PubMed]

34. Martín R, Miquel S, Benevides L, Bridonneau C, Robert V, Hudault S, et al. Functional Characterization of Novel Faecalibacterium prausnitzii Strains Isolated from Healthy Volunteers: A Step Forward in the Use of $F$. prausnitzii as a Next-Generation Probiotic. Front Microbiol. 2017 Jun 30;8:1226. [PubMed]

35. Oberc A, Coombes BK. Convergence of External Crohn's Disease Risk Factors on Intestinal Bacteria. Front Immunol. 2015 Nov 3;6:558. [PubMed]

36. Finegold SM, Molitoris D, Song Y, Liu C, Vaisanen ML, Bolte E, et al. Gastrointestinal microflora studies in late-onset autism. Clin Infect 
Dis. 2002 Sep 1;35(Suppl 1):S6-S16. [PubMed]

37. Song Y, Liu C, Finegold SM. Real-time PCR quantitation of clostridia in feces of autistic children. Appl Environ Microbiol. 2004 Nov;70(11):6459-65. [PubMed]

38. Parracho HM, Bingham MO,
Gibson GR, McCartney AL. Differences between the gut microflora of children with autistic spectrum disorders and that of healthy children. J Med Microbiol. 2005 Oct;54(Pt 10):987991. [PubMed]

39. Finegold SM. Therapy and epi- demiology of autism-clostridial spores as key elements. Med Hypotheses. 2008; 70(3):508-11. [PubMed]

40. Horvath K, Perman JA. Autism and gastrointestinal symptoms. Curr Gastroenterol Rep. 2002 Jun;4(3):2518. [PubMed]

Please cite this article as: Ibryamova S, Petkov V, Ignatova-Ivanova T, Kolev G. Social status and Gut Microbial Dysbiosis in adults and children with Autism Spectrum Disorders: a profound review of the available literature sources. $J$ of IMAB. 2021 Jul-Sep;27(3):3939-3946. DOI: https://doi.org/10.5272/jimab.2021273.3939

Received: 28/03/2021; Published online: 14/09/2021

Address for correspondence:

Tsveteslava Ignatova-Ivanova

Department of Biology, Shumen University "Konstantin Preslavski"

115, Universitetska Str., Shumen, Bulgaria

E-mail: ts.ignatovaivanova@shu.bg 